\title{
Research on the Effect of Sound Velocity Vertical Structure on Sonar Detection Performance in Deep Sea Convergent Zone
}

\author{
Shiyao Lin, Jinhua Hua , Jun Yuan, Wei Zhang \\ School of Electronic Engineering, Naval University of Engineering, Wuhan 430033, China. \\ a18642269056@163.com
}

\begin{abstract}
Keywords: sound convergence zone, sound velocity vertical structure, detection performance,
\end{abstract} performance evaluation, sonar detection.

\begin{abstract}
This article focuses on how to accurately evaluate the influence of sound velocity vertical structure on sonar detection performance under acoustic convergence condition. Combining acoustic propagation model with sonar target detection theory, a quantitative performance evaluation model is constructed which evaluates the sonar detection capability. The effect of vertical structure of sound velocity on the performance is analyzed quantitatively. Finally, the validity of the model is verified by simulation.
\end{abstract}

\section{Introduction}

Acoustic convergence is a common natural phenomenon in the ocean. Acoustic waves transmitted by the sound source near the sea surface bend and reverse in the deep sea, and fold back into the sea several times in areas far from the sound source to form multiple high-intensity zones. With this phenomenon, sonar can detect long-range underwater targets.

The deep-sea sound propagation path is affected by the vertical structure of sound velocity. In order to assess the detection performance of sonar accurately, it is necessary to analyze the effect of changes in the vertical structure of sound velocity on sonar detection performance. Most of the traditional sonar performance analysis methods are based on straight line propagation of sound waves [1.2]. These methods can analyze the detection performance of sonar when the vertical structure of sound velocity changes little. However, it is difficult for these methods to evaluate the sonar detection performance accurately in the case where the vertical structure of the sound velocity changes greatly.

The research on the deep-sea convergence zone mainly focuses on the forming mechanism of the convergence zone and the influence of the vertical structure of sound velocity on the convergence zone. Combining acoustic propagation model with sonar target detection theory, a quantitative performance evaluation model is constructed which evaluates the sonar detection ability. The effect of vertical structure of sound velocity on the performance is analyzed quantitatively.

\section{Sound Velocity Vertical Structure Model}

Typical deep-sea sound velocity profile can be classified from shallow too deep as mixing layer, thermocline, and deep ocean isotherm. Munk model can describe deep-sea sound velocity profile well [3].

$$
F(z)=c_{\mathrm{DSC}}\left[1+\varepsilon\left(\eta+e^{-\eta}-1\right)\right], \eta=2\left(z-z_{\mathrm{DSC}}\right)
$$

Where $z$ is depth; $z_{D S C}$ and $C_{D S C}$ is the depth and the velocity of the channel axis; $\eta$ is the non-dimensional range; $\mathrm{D}$ is the channel thickness; $\varepsilon$ is the disturbance coefficient. In order to control the vertical structure of sound velocity by a given environmental parameter, a linear transformation of (1) is needed to obtain the relationship between sound velocity and depth.

In order to match the calculation result $\mathrm{F}(\mathrm{z})$ of the Munk model with the actual velocity of sound $\mathrm{c}(\mathrm{z})$, following linear transformation should be performed.

$$
\frac{c(z)-c\left(z_{1}\right)}{c\left(z_{2}\right)-c\left(z_{1}\right)}=\frac{F(z)-F\left(z_{2}\right)}{F\left(z_{2}\right)-F\left(z_{1}\right)}
$$


Where the actual velocity of sound at the depth of $\mathrm{z} 1$ and $\mathrm{z} 2$ are $\mathrm{c}(\mathrm{z} 1)$ and $\mathrm{c}(\mathrm{z} 2)$; the sound velocity calculated by the Munk model are $F(z 1)$ and $F(z 2)$.

\section{The Model of Acoustic Propagation}

This article uses the Bellhop model to simulate acoustic propagation in the deep sea. The Bellhop model is a sound propagation model developed by Porter that uses Gaussian rays instead of geometric rays.

The basic ray equations in the cylindrical coordinate system can be expressed as:

$$
\frac{d}{d s}\left[\frac{1}{c(r, z)} \frac{d r}{d s}\right]=-\frac{1}{c^{2}(r, z)} \nabla c(r, z)
$$

Where $r$ is the range; $z$ is the depth; $s$ is the arc length along the sound line; $c$ is the sound velocity.

The constraint variables $p$ and $q$ are introduced to control the energy distribution of the Gaussian beam and a dynamic tracking equation is established [4.5]:

$$
\begin{gathered}
\frac{d q}{d s}=c p(s) \\
\frac{d p}{d s}=-\frac{c_{m}}{c^{2}(s)} q(s)
\end{gathered}
$$

Where $c_{m}$ is the second-order differential perpendicular to the sound ray direction. The sound pressure generated by a single sound line can be expressed as:

$$
P(s, n)=A(s) \phi(n, s) e^{j w t}
$$

Where $n$ is the displacement perpendicular to the center of the sound ray; $\omega$ is frequency; $A$ is amplitude along the sound ray; $\phi$ is the Effect function perpendicular to the direction of sound rays; $\tau$ is the travel time along the sound ray. The sound field is the superposition of all sound lines. Sonar target detection theory

When a sonar operates in the convergence zone, the sonar receiver performance is mainly affected by the noise of the marine environment.

Active sonar equation under noise masking is [6]

$$
S L-2 T L-N L+T S+D I=D T
$$

where $S L$ is the source level of an active sonar; $N L$ is the noise level of ocean environment; $T L$ is the propagation loss; TS is the target strength, DI is the directivity index, and DT is the detection threshold.

The active sonar's receiver detection $D T$ can be expressed by using the equation [7]

$$
D T=10 \lg (S / N)_{\text {in }}=S L-2 T L-N L+T S+D I
$$

Sonar signal processor uses square-law detection. The best receiver performance of detecting signals in Gaussian noise meets the following relationship

$$
(S / N)_{\text {out }}=B \cdot T \cdot(S / N)_{\text {in }}
$$

where $B$ is the receiver's bandwidth, and $T$ is the integration time of an integrator.

In the case of Gaussian noise, when the signal-to-noise ratio is small, the receiver's detection characteristics satisfy the formula

$$
P_{d}=1-G\left(G^{-1}\left(1-P_{f}\right)-(S / N)_{\text {out }}^{1 / 2}\right)
$$

where $P_{d}$ is the detection probability of the system with a false alarm probability which is $P_{f}, G$ is the standard normal distribution function. Equations (8) and (9) are brought into equation (10) to yield equation (11)

$$
P_{d}=1-G\left[G^{-1}\left(1-P_{f}\right)-\left(B \cdot T \cdot 10 \frac{S L-N L-2 T L+T S+D I}{10}\right)^{1 / 2}\right]
$$

Equation (11) is the detection probability of sonar under constant false alarm. 


\section{Sonar Detection Performance Evaluation Model}

Sonar detection performance is mainly measured by the effective detection zone's width and the effective detection range.

The active depth range of underwater targets is approximately from $0 \mathrm{~m}$ to $200 \mathrm{~m}$, and it is significant to evaluate the detection ability of sonar in this range. Assuming that the marine environment around 360 degrees around the surface ship is isotropic. The horizontal range of target is $\mathrm{x} \mathrm{m}$ and the depth are $\mathrm{y} \mathrm{m}$, and the probability of detection is $\mathrm{Pd}(\mathrm{x}, \mathrm{y})$.

When the sonar receives a target echo larger than the minimum detectable signal of the sonar, it is considered that the sonar can detect the target. Based on the detection probability under the constant false alarm probability, the zone with the detection probability greater than 0.5 is set as the effective detection zone.

According to the acoustic propagation model, the width of the convergence zone is different at different depths. In the kth convergence zone, at the depth of $y \mathrm{~m}$, the width of the effective detection zone is $\mathrm{dk}(\mathrm{y})$.

The arithmetic average of the width of the effective detection zone at each depth from $0 \mathrm{~m}$ to 200 $m$ is taken as the effective detection zone width of the kth convergence zone:

$$
d_{k}=\bar{d}_{k}(y)
$$

Surface ships always want to find underwater targets as far as possible, which will give the surface warships enough time for the next action.

Take the arithmetic average of the effective detection zone range at each depth from $0 \mathrm{~m}$ to 200 $\mathrm{m}$ as the effective detection range of the kth convergence zone:

$$
D_{k}=\bar{D}_{k}(y)
$$

In the ocean at the same latitude, the sea surface temperature varies greatly in different seasons, but the depth of the channel axis and the temperature of the channel axis are relatively stable and do not change with the season. When the sea surface temperature changes, the water shallower than the channel axis changes its temperature. Changes in surface water temperature will change the vertical structure of sound velocity above the channel axis.

The wind and waves stir the seawater on the surface of the ocean to form a mixing layer. The rising wind will make the mixing layer thicker. As the thickness of the mixing layer changes, the upper boundary of the thermocline changes. Variations in the thickness of the mixing layer change the vertical structure of the sound velocity above the channel axis.

The change of the vertical structure of the sound velocity have a great influence on the sound propagation path, thus affecting the ability of the sonar to detect the target in the convergence zone.

\section{Simulation Analysis}

Assuming that the salinity is the same throughout the ocean, the vertical structure of sound velocity is only affected by temperature and depth. Set the sea surface temperature to 22 degrees Celsius and the mixing layer thickness is $50 \mathrm{~m}$. The lower boundary of the mixing layer is the upper boundary of the thermocline. The lower boundary of the thermocline is $400 \mathrm{~m}$, and the temperature is always 8 degrees Celsius. The depth of the channel axis is $1100 \mathrm{~m}$, and the temperature is always 4 degrees Celsius. Sonar parameters set to typical values. The false alarm probability is 0.01 . The distribution of detection probability is shown in Fig. 1. 


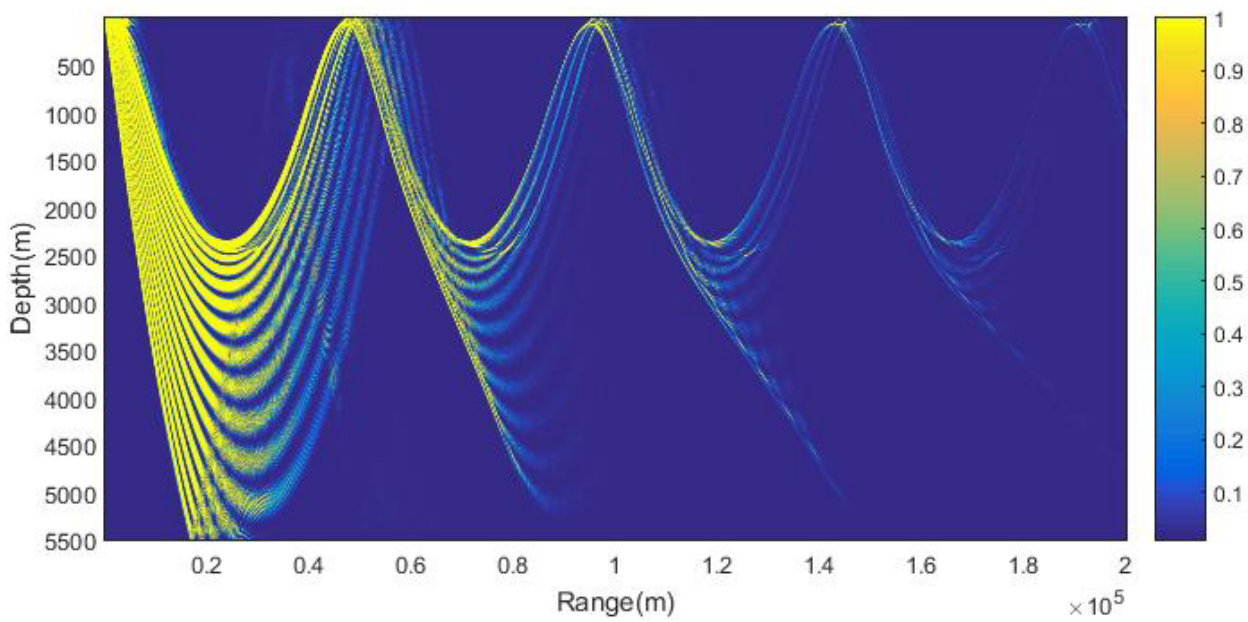

Fig 1. The distribution of detection probability

The simulation results show that sound waves transmitted by the sound source near the sea surface are bent and reversed in the deep sea and are folded back to the sea surface several times away from the sound source to form multiple high sound power intensity zones. These zones are convergence zones. In the convergence zone, sonar can detect underwater targets effectively.

Assuming that the ocean surface water temperature increases from 15 degrees Celsius to 30 degrees Celsius, the ocean water temperature above the channel axis will increase. However, neither the depth of the channel axis nor the water temperature changes. The effective detection range of the first convergence zone and the second convergence zone change with the changing temperature as shown in the Fig. 2. The effective detection width of the first convergence zone and the second convergence zone change with the changing temperature as shown in the Fig. 3.

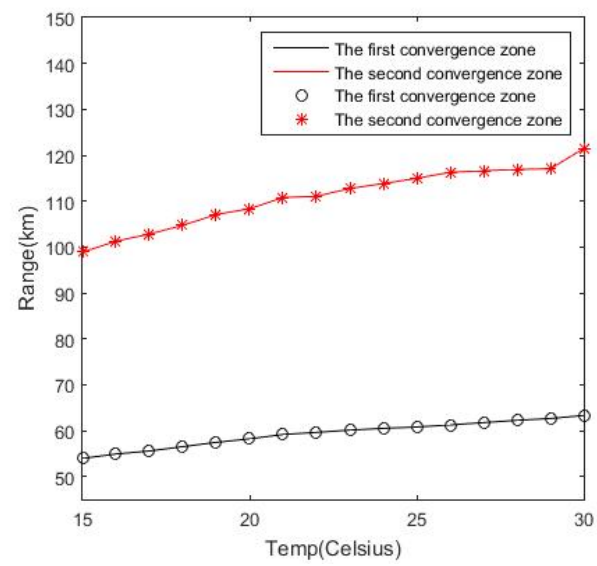

Fig 2. The range of the effective detection zone changes temperature

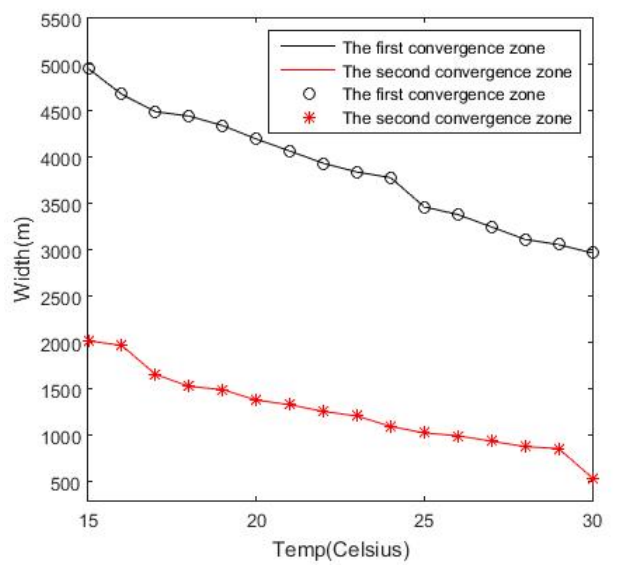

Fig 3. The width of effective detection zone changes with temperature

Let the ocean surface water temperature be 17.4 degrees Celsius (the average temperature of the global ocean surface), and the mixing layer thickness increase from $0 \mathrm{~m}$ to $70 \mathrm{~m}$. The effective detection range of the first convergence zone and the second convergence zone change with the changing mixing layer thickness as shown in the Fig. 4. The effective detection width of the first convergence zone and the second convergence zone change with the changing mixing layer thickness as shown in the Fig. 5. 


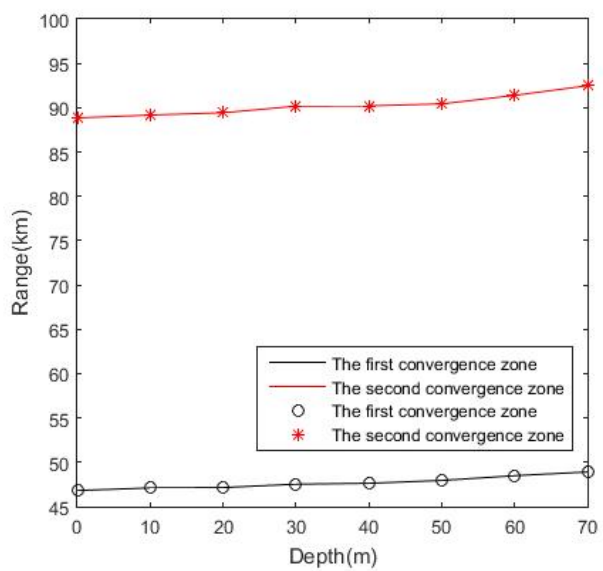

Fig 4. The range of the effective detection zone changes with mixing layer thickness

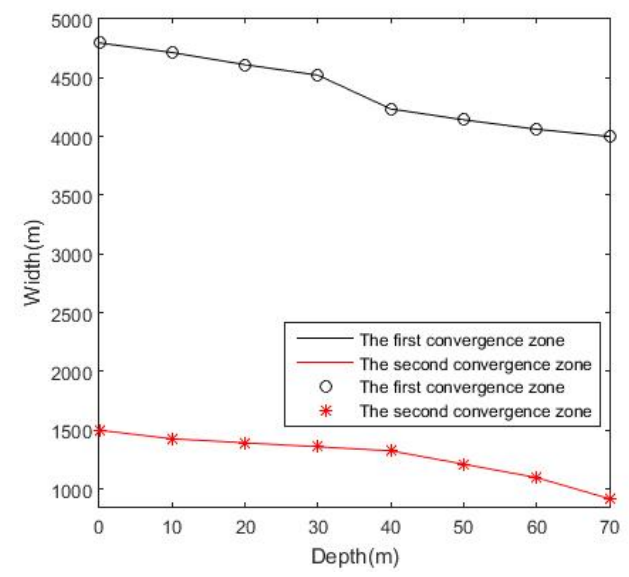

Fig 5. The widthof the effective detection zone changes with mixing layer thickness

The simulation results show that with the increase of sea surface water temperature and the increase of the mixing layer thickness, the effective detection ranges of both the first convergence zone and the second convergence zone is improved, and the improving speed of the second convergence zone is faster than that of the first convergence zone. The effective detection widths of both the first convergence zone and the second convergence zone both decrease, and the falling speed of the first convergence zone is faster than that of the second convergence zone.

The sea surface temperature rose 1 degree Celsius, the range of the first convergence zone increased approximate $624.1 \mathrm{~m}$, the width decreased approximate $132.7 \mathrm{~m}$, the range of the second convergence zone increased approximate $1503.4 \mathrm{~m}$, and the width decreased approximate $98.7 \mathrm{~m}$. The mixing layer thickness increases $10 \mathrm{~m}$. The range of the first convergence zone increases approximate $374.2 \mathrm{~m}$ and the width decreases approximate $79.7 \mathrm{~m}$. The range of the second convergence zone increases approximate $860.8 \mathrm{~m}$ and the width decreases approximate $58.3 \mathrm{~m}$.

Surface water temperature and mixing layer thickness affect the deep-sea sound velocity vertical structure directly. Higher surface water temperatures and deeper mixing layer thicknesses increase the thermocline transition strength. An increase in the thermocline transition intensity will result in a larger deflection of the near-field sound line, which will bring the convergence zone closer to the sound source. However, at the same time, the incident angle of the sound ray into the deep sea is also increased, which will make the sound ray get larger horizontal displacement in the process of deep sea return. The effect of deep seawater on sound line deflection exceeds that of the near field greatly. Therefore, the higher the thermocline intensity, the farther the convergence zone is from the sound source. The farther an acoustic wave travels in the ocean, the greater its propagation loss and the less concentrated energy in the convergence zone, resulting in a narrower effective detection width.

\section{Conclusion}

In order to evaluate the influence of the vertical structure of sound velocity on sonar detection performance under acoustic convergence conditions accurately, combining the marine acoustic propagation model with the sonar target detection theory, a quantitative evaluation model of target performance in the sonar detection convergence zone is constructed. The ability of sonar to detect targets in the convergence zone can be measured by the effective detection range and the width of the effective detection zone. Based on it, the influence of the vertical structure of sound velocity on the detection performance is analyzed quantitatively. Simulation results shows.

\section{References}

[1]. Douglas A. Abraham, Peter K. Willett, Active Sonar Detection in Shallow Water Using the Page Test, IEEE JOURNAL OF OCEANIC ENGINEERING, Vol. 27(2002) No.1, p. 35-46. 
[2]. LI Fan, GUO Shengming, WANG Lujun, LIU Qingyu, A new approach to prediction of sonar detection range index, Technical Acoustics, Vol. 28(2009), No.3 p. 235-239.

[3]. Walter H. Munk, Sound channel in an exponentially stratified ocean, with application to SOFAR, J. Acoustic. Soc. Am, Vol. 55(1974), No.2, p. 220-226.

[4]. V. Cerveny, M. M. Popov, I. Psencik, Computation of wave fields in in homogeneous media Gaussian beam approach, Geophys. J. R. Astron. Soc, (1982) p. 109-128.

[5]. Michael B. Porter, Homer P. Bucker, Gaussian beam tracking for computing ocean acoustic fields, J. Acoustic. Soc. Am, Vol. 82(1987), No. 4, p. 1349-1359.

[6]. R. J. Urick, Principles of Underwater Sound. New York: McGraw-Hill.1983.

[7]. Gao Xueqiang, Yang Rijie, Zhou Xu, Simulation Research on the Effect of Thermocline on Active Sonar Searching Submarine, JOURNAL OF TEST AND MEASURMENT TECHNOLOGY, Vol. 22(2008.), No.1, p. 55-58. 